\title{
Changes in Schedule II oral opioid volume dispensed in a private health plan following Florida's Acute Pain Opioid Restriction Law
}

\author{
Razanne Oueini, MSc; Amie Goodin, PhD, MPP; Scott M Vouri, PharmD, PhD; Haesuk Park, PhD; \\ Wei-Hsuan Lo-Ciganic, MSPharm, MS, PhD; and Juan M Hincapie-Castillo, PharmD, MS, PhD
}

\section{What is already known about this subject}

- Over 30 states have recently enacted policies aimed to limit days supply or morphine milligram equivalents (MMEs) of opioid prescriptions.

- In 2018, the state of Florida enacted legislation (House Bill 21) that restricted days supply of Schedule II opioids to 3 days for patients with acute pain.

- Individual health plans and payers have also imposed opioid supply restrictions for acute pain and/or other indications.
What this study adds

- Among enrollees of a single large health plan in Florida, the total units and MMEs dispensed of Schedule II opioids did not decrease significantly following the state policy change.

- This study suggests that the restriction on days supply imposed by the legislation contributed slightly to an already declining trend in use of Schedule II oral opioids.

\section{Author affiliations}

Razanne Oueini, MSc, Department of Pharmaceutical Outcomes \& Policy, University of Florida, Gainesville. Amie Goodin, PhD, MPP; Haesuk Park, PhD; and Wei-Hsuan Lo-Ciganic, MSPharm, MS, PhD, Department of Pharmaceutical Outcomes \& Policy and Center for Drug Evaluation and Safety, University of Florida, Gainesville. Scott M Vouri, PharmD, PhD, Department of Pharmaceutical Outcomes \& Policy; Center for Drug Evaluation and Safety; and UF Health Physicians, University of Florida, Gainesville. Juan M HincapieCastillo, PharmD, MS, PhD, Department of Pharmaceutical Outcomes \& Policy; Center for Drug Evaluation and Safety; and Pain Research and Intervention Center of Excellence, University of Florida, Gainesville.

AUTHOR CORRESPONDENCE:

Juan M Hincapie-Castillo, 352.273.5526;

j.hincapie@ufl.edu

J Manag Care Spec Pharm 2021;27(6):779-84

Copyright $(2021$, Academy of Managed Care Pharmacy. All rights reserved.

morphine milligram equivalent (MMEs) dispensed per month.

METHODS: Pharmacy claims from July 2017 to June 2019 were analyzed from a private health plan serving a large Florida employer. We summed the number of units and the total MMEs dispensed for each month per \author{
1,000 enrollees. Units were defined as the
}

volume dispensed at the plan level remains unknown.

OBJECTIVES: To assess the impact of HB21 on the total volume dispensed of oral Schedule II opioids. We evaluated the change from before to after the law's implementation in (1) total number of opioid units dispensed per month and (2) total
(HB21), implemented into law on July 1 , 2018, limited opioid prescriptions for acute pain to a 3-day supply. While the law has been associated with a decrease in opioid prescribing for acute pain, its effect on opioid 
total quantity of tablets/capsules dispensed for each Schedule II oral opioid prescription. We used interrupted time series (ITS) models, accounting for autocorrelation, to determine any immediate change after the policy implementation and to estimate trends before and after the policy.

RESULTS: We identified 16,226 prescriptions of oral Scheduled II opioids dispensed to 6,315 enrollees over a 2-year period. The HB21 law was associated with an immediate but not statistically significant decrease of 110.25 units dispensed per 1,000 enrollees in the month after implementation $(95 \% \mathrm{Cl}:-218.84,-1.67 ; P=0.06)$. There was an immediate but not statistically significant decrease of 1,456.29 MMEs dispensed per 1,000 enrollees following HB21 implementation ( $95 \%$ $\mathrm{Cl}:-2,983.87,71.29 ; P=0.07)$. There were no significant changes in the slopes of the trends for total number of opioid units and total MMEs dispensed after HB21.

CONCLUSIONS: Despite substantial lower quantities, there were no significant immediate reductions in total opioid units and MMEs dispensed in the year following the implementation of HB21. Our findings can inform other health plans on the potential effect of such restrictive laws and policies in other states where preexisting declining trends might have a higher impact than restriction policies. Future studies are needed to evaluate long-term intended and unintended consequences, including effects on patients' access to care, resulting from this type of restrictive law.

In response to the opioid epidemic in the United States, 39 states have enacted policies limiting the days supply or morphine milligram equivalents (MMEs) of opioid prescriptions by the end of 2019.1-3 Policy evaluations of the different restrictions from these state laws have resulted in mixed findings thus far, with evidence suggesting that meaningful decreases in prescription opioid supply is only observable when the strictest restrictions are implemented., ${ }^{4,5}$ The state of Florida enacted House Bill 21 (HB21), which went into effect on July 1, 2018, restricting prescriptions for Schedule II opioids to 3 days for patients with acute pain. ${ }^{6}$ Prescribers are allowed to write up to a 7-day supply if they document the occurrence as an "acute pain exception." While the HB21 law did not place new limits on the days supply of Schedule III opioid medications or for nonacute pain prescriptions, there were concerns raised regarding the possible unanticipated consequences of the policy on patients in treatment for chronic pain or for severe episodes of acute pain (eg, trauma, postsurgical care).

Our recent analysis of the effect of the HB21 implementation among patients with acute pain in a single large health plan in Florida found significant reductions in opioid use after accounting for declining secular trends before the policy change.7,8 We found a significant immediate decrease in the mean days supply per opioid prescription after the law was implemented. There was also a sustained decreasing trend over the following 8 months after policy implementation of days supplied.

Although the explicit intent of the law was to reduce the days supply of the prescription, our evaluation also indicated that the number of new monthly opioid users decreased significantly from 5.5 to 4.6 patients per 1,000 enrollees per month immediately following the policy change (95\% CI: $-1.53,-0.31$ new users per 1,000 enrollees per month; $P=0.005$ ), suggesting that fewer patients overall were initiated on opioid therapies. These changes were observed even with the plan's restrictions implemented along with HB21, which limited the supply of opioids regardless of the Schedule to 7 days. Additional reports from single institutions in Florida have found that the HB21 law was associated with significant decreases in the percentage of patients prescribed opioids and the mean total opioid dose prescribed at discharge from surgery. ${ }^{9,10}$

With evidence of decreased opioid use from changes in prescribing practices in the acute pain setting, it is necessary to consider the consequences of restriction policies such as HB21 on health plans at large. Having evidence of the payer-wide effects of these restriction policies can help inform those plans in states for which no legal mandate exists yet or for plans across the country that could be subject to federal-level restrictions. While some health plans have introduced individual opioid prescribing restrictions in reaction to the US opioid crisis, to date no investigation has evaluated the effect of statewide mandates on opioid restrictions across all plan enrollees.

This study aimed to assess the impact of restrictions placed by the Florida HB21 law on the total volume of Schedule II oral opioids dispensed to enrollees within a single private large health plan in Florida. We hypothesized that, similarly to the effects seen for the lower quantity of opioids dispensed for acute pain patients, the law was associated with a significant immediate decrease in the total volume of opioids dispensed for all plan enrollees as measured by total units and total MMEs dispensed.

\section{Methods}

\section{DATA SOURCES}

We analyzed pharmacy prescription claims dispensed from July 1, 2017, to June 30, 2019, encompassing the 1 year before and after the implementation of HB21 on July 1, 2018. Claims data were obtained from a single health plan that serves over 45,000 employees of a large university and health 


\section{FIGURE 1 Total Units Dispensed of Schedule II Oral Opioids per 1,000 Plan Enrollees Before and After Implementation of Florida House Bill 21 on July 1, 2018}

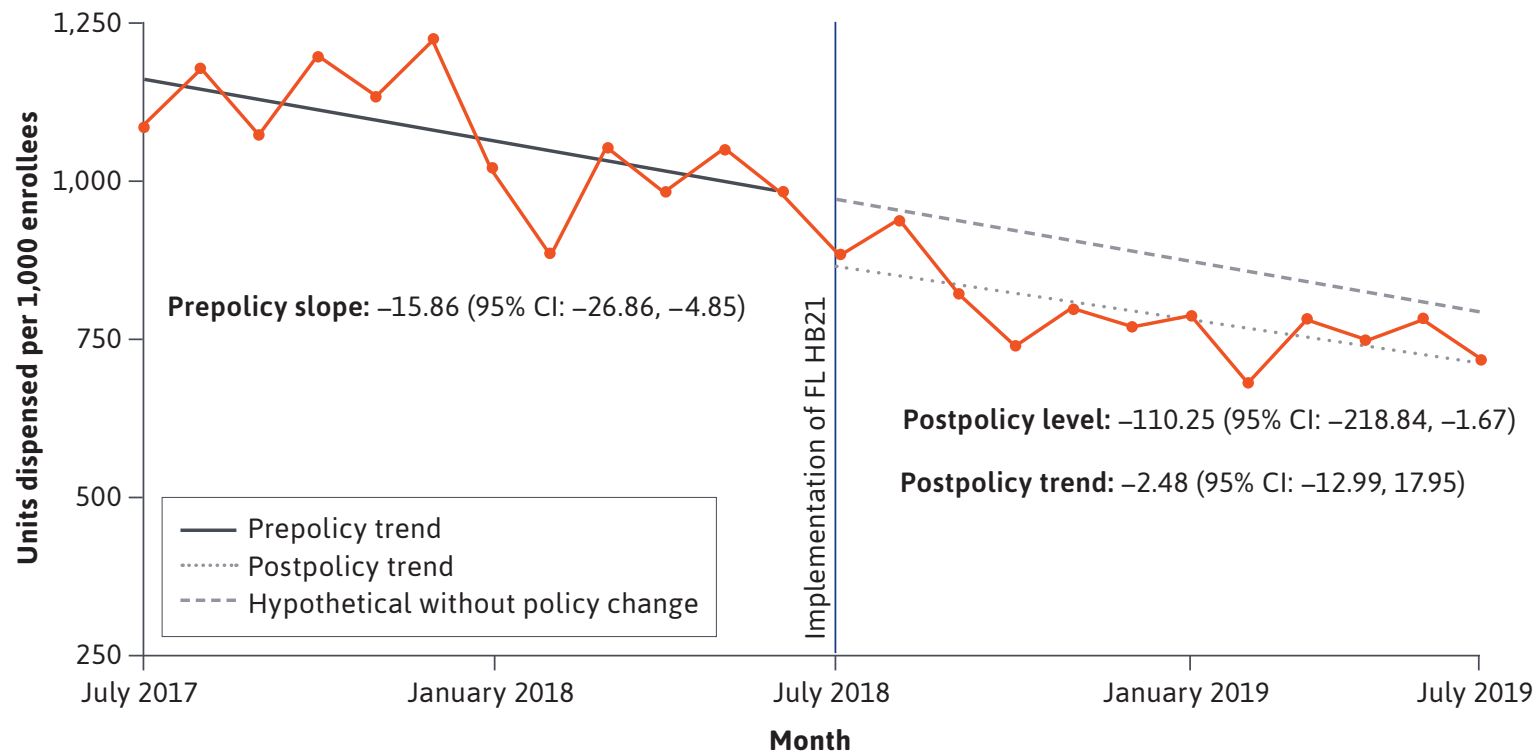

FL HB21 = Florida House Bill 21; $M M E=$ morphine milligram equivalent .

system employer in Florida. We included single entity and combination products of Schedule II oral opioids indicated for pain management.

\section{MEASURES}

We calculated the following monthly measures for prescriptions of oral Schedule II opioids: (1) total number of opioid units dispensed per 1,000 enrollees and (2) total MMEs dispensed per 1,000 enrollees. Units were defined as the total quantity of tablets or capsules dispensed for each prescription. MMEs were calculated using the conversion factors published by the Centers for Disease Control and Prevention. ${ }^{11}$ While the language of the law explicitly required a reduction in the number of days supply for the opioid prescriptions, there were no stipulations that limited individual units and MMEs allowed. The selection of these 2 measures allowed for a more complete analysis of the potential unintended effects on prescribing behavior.

\section{STATISTICAL ANALYSES}

Monthly measures were analyzed using interrupted time series (ITS) models that accounted for autocorrelation of error terms. Our ITS models were used to determine any immediate change following the implementation of HB21 and to estimate trends before and after the policy. For policy analyses, ITS is the preferred quasi-experimental study design, since it allows for an assessment of preexisting trends in the data before implementation (ie, time effect) and immediate changes in the outcome (ie, level effect) and changes in the outcome trend after the interruption (ie, trend effect). ${ }^{12}$ Using the "nlme" $\mathrm{R}$ package, we fitted generalized least squares linear models with an autoregressive-moving average correlation structure of order $(\mathrm{p}, \mathrm{q})$. The $\mathrm{p}$ and $\mathrm{q}$ parameters were obtained from analyzing the autocorrelation and partial autocorrelation functions of each time series. A 2 -sided $\mathrm{P}<0.05$ was considered statistically significant in evaluating the model coefficients for the time effect, and the level and trend changes resulting from the policy interruption. All analyses were conducted with R, version 4.0.3 (R Foundation for Statistical Computing) and SAS, version 9.4 (SAS Institute). This study received approval from the institutional review board at the University of Florida.

\section{Results}

We analyzed 16,226 prescriptions of oral Scheduled II opioids dispensed to 6,315 enrollees in a private health plan from July 1, 2017, to June 30, 2019. 


\section{FIGURE 2 Total MME Dispensed of Schedule II Oral Opioids per 1,000 Plan Enrollees Before and After Implementation of Florida House Bill 21 on July 1, 2018}

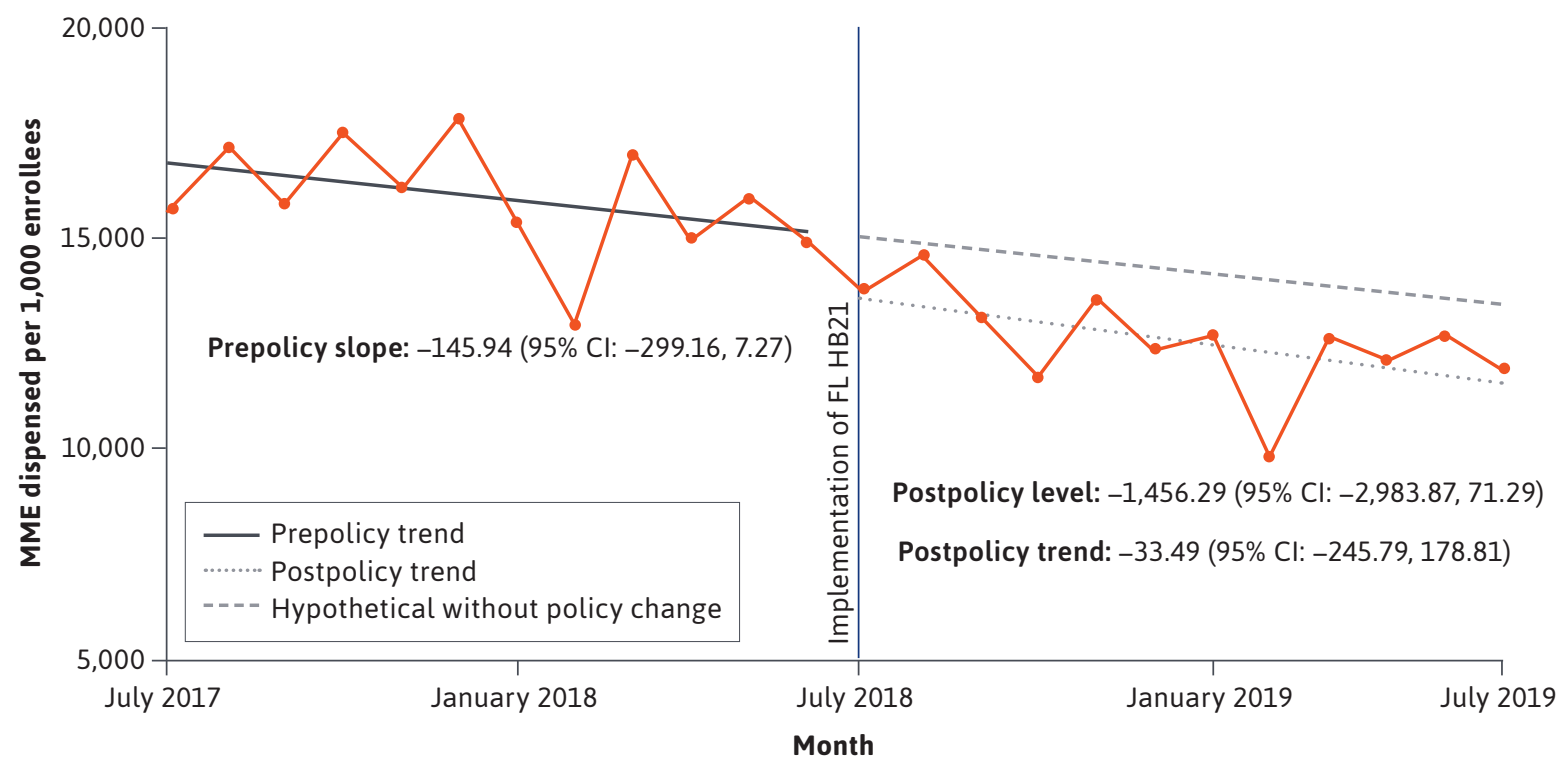

FL HB21 = Florida House Bill 21; MME = morphine milligram equivalent.

\section{CHANGES IN TOTAL NUMBER OF OPIOID UNITS DISPENSED}

During the preimplementation period of July 1, 2017, to June 30,2018 , there was a significant decline in the total number of units of Schedule II oral opioids dispensed per 1,000 plan enrollees (-15.86 units per month; 95\% CI: $-26.86,-4.85$; $\mathrm{P}=0.01$; Figure 1). Compared with data from the previous year, and accounting for this preexisting declining trend, the implementation of the HB21 law was associated with an immediate but not statistically significant decrease of 110.25 units dispensed per 1,000 plan enrollees (95\% CI: -218.84, $-1.67 ; \mathrm{P}=0.06)$. There was not a statistically significant change in the postpolicy trend in the year following implementation of HB21 (July 1, 2018-June 30, 2019; -2.48 units per 1,000 enrollees per month, 95\%CI: $-12.99,17.95 ; \mathrm{P}=0.76$ ).

\section{CHANGES IN TOTAL MMES DISPENSED}

In the year preceding HB21 implementation, the MMEs for Schedule II opioids dispensed per 1,000 enrollees decreased at a rate of 145.94 MMEs per month (95\% CI: -299.16, 7.27; $\mathrm{P}=0.07$; Figure 2). There was an immediate decrease of 1,456.29 MMEs dispensed per 1,000 enrollees in following month after HB21 implementation (95\% CI -2,983.87, 71.29; $P=0.07)$. There was no significant change in the slope of the trend for total MMEs dispensed in the period after implementation (-33.49 MME per 1,000 per month, 95\% CI: $-245.79,178.81 ; \mathrm{P}=0.76)$.

\section{Discussion}

This study documents substantial but not significant reductions in the total number of units and MMEs of Schedule II oral opioids dispensed among enrollees of a single large health plan in Florida following the implementation of the HB21 opioid restriction law on July 1, 2018. Based on our findings, the only significant decreases measured were the number of monthly units dispensed across the entire study period, which spanned from July 1, 2017, to June 30, 2019. The lack of significant immediate reductions (ie, level changes) despite substantial lower quantities of opioids dispensed measured by the total number of units and the total MMEs of the prescriptions might be explained by the steep declining trends before implementation. The rate of change of monthly units and MMEs dispensed per month did not differ significantly from the prepolicy period, which could support the evidence of a strong effect of these secular trends that have been documented nationwide. National estimates of opioid prescribing has shown sustained decreases since a peak in $2012 .^{13}$ 
Our findings add to the growing body of knowledge regarding the consequences of state-level opioid restriction policies across the United States. While opioid supply restriction laws are intended to mitigate the potential for opioid misuse, abuse, and diversion, the country continues to grapple with increasing rates of opioid-related deaths despite such legislation..$^{14}$ These policies have been criticized for potential unanticipated consequences for pain patients, such as reduced access to opioid pharmacotherapies, therapy discontinuity, and decreased willingness for primary care providers to accept new chronic pain patients. ${ }^{15-17}$ In addition, these unanticipated consequences can disproportionally affect vulnerable patients, such as those insured by Medicaid programs that already experience access barriers to proper pain management. ${ }^{18,19}$

While opioid restriction policies have contributed to marked decreases in use, pain professionals and advocacy groups are arguing for a more calibrated approach that would guarantee appropriate pain management and access to analgesia. ${ }^{20}$ Recently, 2 class action lawsuits have been filed against large chain pharmacies for discriminatory practices in refusing to fill opioid medications for treatment as recommended via prescription..$^{21,22}$ The plaintiffs argue that these companies have violated the Americans with Disability Act, the Rehabilitation Act, and the Affordable Care Act.

The effects seen for Florida HB21 can inform policy development in other states seeking to limit opioid use, as well as in the federal government. The John S. McCain Opioid Addiction Prevention Act, a bipartisan federal bill introduced in 2019 in the US Senate (but later rescinded), sought to enforce a limit of 7 days supply on Schedule II opioid prescriptions for acute pain. ${ }^{23}$ While this bill included specifications similar to those of Florida HB21 with regards to opioid prescriptions that are excluded from restrictions (eg, opioids prescribed for chronic pain, cancer pain, hospice and end-of-life care, and palliative care), there was concern that unanticipated consequences of this legislation would have affected patient access to care. Future legislation should be responsive to avoiding potential harm to patients experiencing pain, and health plans must work to ensure that appropriate care is provided to beneficiaries in light of prescribing restrictions.

\section{LIMITATIONS}

This study has several limitations that should be considered in the interpretation and application of our findings. First, the data used for this study originated from a single, employer-sponsored health plan consisting of a population that is relatively younger and healthier than typical opioid initiators. Our findings may therefore be less applicable to populations that are uninsured or receiving coverage through government payers with managed care plans or state-administered Medicaid health plans.

Second, the health plan in this study also enacted pharmacy-level opioids policy "edits," which were implemented by the pharmacy benefits manager in conjunction with the HB21 policy, where edits are defined by pharmacy benefits managers as conditions to be satisfied in order to receive coverage for medical goods and services. With this particular edit, patients in the health plan were limited to a 7-day supply of opioids regardless of the Schedule. In addition, a prior authorization was required if a subsequent opioid was attempted to be filled within 29 days of the previous opioid. This policy change may have also contributed to the decreased Schedule II oral opioid use that we reported. Nevertheless, it is important to point out that HB21 was stricter in its restrictions for opioid supply than other plan edits.

Third, the HB21 law mandated that prescribing practitioners consult Florida's Prescription Drug Monitoring Program (PDMP) before prescribing controlled substances. Our analysis cannot disentangle whether the effects of the policy change were driven primarily by the law's restriction on opioid prescribing or by requiring prescribers to consult the PDMP.

Finally, this study did not evaluate long-term intended and unanticipated consequences of HB21, such as changes in the dispensing of other non-Schedule II opioids or other adjuvant medications. Such consequences should also be evaluated to inform future policy.

\section{Conclusions}

In the 1 year following implementation of Florida HB21, there were substantial but not statistically significant decreases in the total units and MMEs of Schedule II oral opioids dispensed to enrollees of 1 large employer-sponsored health plan in the state. This evidence can inform other health systems and private health plans on the potential effects of similar policies restricting prescribing. Additional research is needed to assess other intended and unanticipated consequences of this legislation, specifically those dealing with health outcomes and service utilization for pain patients whose access to care may be affected. 


\section{DISCLOSURES}

No outside funding supported this study. The authors report no conflicts of interest.

Preliminary results of this study were presented at the Virtual ISPOR 2020 Conference held May 18-20, 2020.

\section{REFERENCES}

1. Davis CS, Lieberman AJ, HernandezDelgado H, Suba C. Laws limiting the prescribing or dispensing of opioids for acute pain in the United States: a national systematic legal review. Drug Alcohol Depend. 2019;194:166-72.

2. National Conference of State Legislatures. Prescribing policies: states confront opioid overdose epidemic. June 30, 2019. Accessed July 1, 2019. http://www.ncsl.org/research/health/ prescribing-policies-states-confrontopioid-overdose-epidemic.aspx

3. Davis CS, Lieberman AJ. Laws limiting prescribing and dispensing of opioids in the United States, 1989-2019. Addiction. Published online November 27, 2020. doi: 10.1111/add.15359.

4. Chua K-P, Kimmel L, Brummett CM. Disappointing early results from opioid prescribing limits for acute pain. JAMA Surg. 2020;155(5):375-76.

5. Dave CV, Patorno E, Franklin JM, et al. Impact of state laws restricting opioid duration on characteristics of new opioid prescriptions. J Gen Intern Med. 2019;34(11):2339-41.

6. The Florida Senate. 2018 Florida Statutes. FLA. STAT. § 456.44(1) (2018). Accessed May 6, 2021. https://www. flsenate.gov/laws/statutes/2018/456.44

7. Hincapie-Castillo JM, Goodin A, Possinger M-C, Usmani SA, Vouri SM. Changes in opioid use after Florida's restriction law for acute pain prescriptions. JAMA Netw Open. 2020;3(2):e200234.
8. Hincapie-Castillo J, Goodin A, Vouri S, Lo-Ciganic W, Park H. PDG33 Changes in Schedule II oral opioid volume dispensed in a private payer following Florida's acute pain opioid restriction law [abstract]. Value Health. 2020;23(Suppl 1):S135.

9. Porter SB, Glasgow AE, Yao X, Habermann EB. Association of Florida House Bill 21 with postoperative opioid prescribing for acute pain at a single institution. JAMA Surg. 2020;155(3):263-64.

10. Potnuru P, Dudaryk R, Gebhard RE, et al. Opioid prescriptions for acute pain after outpatient surgery at a large public university-affiliated hospital: impact of state legislation in Florida. Surgery. 2019;166(3):375-79.

11. Centers for Medicare \& Medicaid Services. Opioid oral morphine milligram equivalent (MME) conversion factors. 2017. Accessed January 3, 2019. https://www. cms.gov/Medicare/Prescription-DrugCoverage/PrescriptionDrugCovContra/ Downloads/Opioid-Morphine-EQConversion-Factors-Aug-2017.pdf

12. Shadish WR, Cook TD, Campbell DT. Quasi-experiments: interrupted timeseries designs. In: Shadish, WR, Cook TD, Campbell DT. Experimental and QuasiExperimental Designs for Generalized Causal Inference. 2d ed. Houghton Mifflin; 2002:171-245.

13. Guy GP, Zhang K, Schieber LZ, Young R, Dowell D. County-level opioid prescribing in the United States, 2015 and 2017. JAMA Intern Med. 2019;179(4):574-76.

14. Wilson N, Kariisa M, Seth P, Smith H, Davis NL. Drug and Opioid-Involved Overdose Deaths - United States, 20172018. MMWR Morb Mortal Wkly Rep. 2020;69(11):290-97.

15. Lagisetty PA, Healy N, Garpestad C, Jannausch M, Tipirneni R, Bohnert ASB. Access to primary care clinics for patients with chronic pain receiving opioids. JAMA Netw Open. 2019;2(7):e196928.
16. Alford DP. Opioid prescribing for chronic pain-achieving the right balance through education. N Engl J Med. 2016;374(4):301-03.

17. Kertesz SG, Gordon AJ. A crisis of opioids and the limits of prescription control: United States. Addiction. 2019;114(1):169-80.

18. Mikosz CA, Zhang K, Haegerich T, et al. Indication-specific opioid prescribing for US patients with Medicaid or private insurance, 2017. JAMA Netw Open. 2020;3(5):e204514.

19. Janakiram C, Fontelo P, Huser V, et al. Opioid prescriptions for acute and chronic pain management among Medicaid beneficiaries. Am J Prev Med. 2019;57(3):365-73.

20. Haffajee RL. The public health value of opioid litigation. J Law Med Ethics. 2020;48(2):279-92.

21. Smith v. Walgreens Boots Alliance, Inc., 2021 U.S. Dist. LEXIS 21734, 2021 WL 391308 (United States District Court for the Northern District of California February 3, 2021, Filed). Accessed May 6, 2021. https://advance.lexis.com/api/ document?collection=cases\&id=urn:con tentItem:61XR-Y121-JPGX-S2J9-0000000\&context $=1516831$

22. Edith Fuog and Those Similarly Situated v. CVS Pharmacy, INC; CARMARK PHC, LLC. United States District Court District of Rhode Island. 2020. Accessed May 6, 2021. https://www.wfla.com/ wp-content/uploads/sites/71/2020/08/ Class-Action-Complaint-Fuog-vs-CVSPharmacy.pdf

23. John S. McCain Opioid Addiction Prevention Act. S.724, 116th Cong. (2019). Accessed May 6, 2021. https://www.govtrack.us/congress/bills/116/s724 